\title{
Metabolic syndrome and its components associated with endothelial dysfunction in chronic kidney disease patients
}

This article was published in the following Dove Press journal:

Vascular Health and Risk Management

5 January 2012

Number of times this article has been viewed

\section{Qiong Bai \\ Xuan Lai \\ Ai-Hua Zhang \\ Xin-Hong Lu \\ Shun-Li Tian \\ Min-Hua Fan \\ Yue Wang \\ Tao Wang}

Division of Nephrology, Peking University Third Hospital, Beijing,

People's Republic of China
Correspondence: Ai-Hua Zhang Division of Nephrology,

Peking University Third Hospital, Beijing, People's Republic of China

Tel +86 I36 4I 277024

Email rosezhang998@hotmail.com
Background: Cardiovascular disease is more common in patients with chronic kidney disease (CKD) than in the general population. Endothelial dysfunction is an early predictor of cardiovascular events.

Objective: We conducted a cross-sectional study in CKD patients to explore the association of metabolic syndrome (MetS) components with endothelial cell function.

Methods: We evaluated clinical and laboratory data in $161 \mathrm{CKD}$ patients from stage 1 to stage 5 . Endothelial function was estimated by flow-mediated dilatation (FMD) of the brachial artery and expressed as percentage change relative to baseline diameter. MetS was defined according to the National Cholesterol Education Program-Adult Treatment Panel III criteria.

Results: Patients were grouped into two groups according to whether or not they had MetS. FMD was significantly lower in the MetS group than in the group without MetS $(P=0.012)$. In a Pearson's correlation analysis, FMD was significantly negatively correlated with waist circumference in women $(r=-0.223, P=0.03)$ and fasting blood glucose $(r=-0.186, P=0.001)$. Multiple linear regression analysis showed that fasting blood glucose was an independently associated factor for FMD.

Conclusion: MetS and some components of MetS (waist circumference in women and fasting blood glucose) are closely associated with a decreased FMD in CKD patients.

Keywords: metabolic syndrome, endothelial dysfunction, flow-mediated dilatation, chronic kidney disease

\section{Introduction}

Metabolic syndrome (MetS) is a cluster of metabolic abnormalities, including visceral obesity, high blood pressure, hyperglycemia, and dyslipidemia, ${ }^{1-4}$ which are associated with increased cardiovascular risk in various clinical conditions. ${ }^{5,6}$ The mechanisms through which this syndrome increases cardiovascular risk are only partially understood. Recently, research has found that several of the individual components of MetS, including high blood pressure, ${ }^{7}$ hyperglycemia, ${ }^{8}$ and abdominal obesity, ${ }^{9,10}$ have been related to increased endothelial dysfunction in the general population.

Endothelial dysfunction, detected noninvasively as a decreased flow-mediated dilation (FMD) of the brachial artery, ${ }^{11,12}$ is an early and potentially reversible functional disturbance in the development of vascular lesion and disease. ${ }^{13}$ It is also common in subjects with MetS, and it is believed to reflect the metabolic derangements present in this condition. ${ }^{14}$

In CKD, endothelial dysfunction also appears to predict cardiovascular disease (CVD) and outcome. ${ }^{15}$ Johnson et $\mathrm{al}^{16}$ have shown that MetS is exceedingly common 
in CKD, and it is thus also a plausible cause of high morbidity and mortality. To date, the relationship of MetS to endothelial dysfunction has been evaluated mostly in the general population but is not clearly elucidated in the CKD population. In the present study, we aimed to investigate the possible link of metabolic components of MetS of patients with CKD with endothelial function assessed by FMD.

\section{Subjects and methods Subjects and collection of data}

This study was approved by the Ethics Committee of Peking University Third Hospital, Beijing, People's Republic of China, and all patients gave written informed consent to all the procedures.

All patients visiting our CKD outpatient clinics from December 1, 2008 to June 30, 2010 were eligible to participate in this study except for those with (1) clinical or laboratory evidence of heart failure (New York Heart Association classes III-IV); (2) peripheral arterial disease; (3) preexistent chronic inflammatory disease; (4) cancer; (5) active arteriovenous fistula, which may interfere with the results of FMD; and (6) primary or secondary nephrotic syndrome. A total of 161 individuals ( 78 men and 83 women; age range 20-88 years; mean $65.58 \pm 14.57$ years) were studied. All patients' clinical data and laboratory test data were collected. A dedicated physician and nurse were responsible for all blood pressure, weight, and waist circumference measurements and performed measurements under strict instructions without any knowledge of the study protocol or objectives. The waist circumference measurement point in this study was the umbilical level. The mercury sphygmomanometer was calibrated regularly. The patients were allowed to take regular antihypertensive medications before each visit. The antihypertensive medications prescribed during the previous days were recorded in detail to calculate the defined daily dose. ${ }^{17}$ Moreover, every drug agent, such as angiotensin-converting enzyme inhibitors (ACEIs) and angiotensin receptor blockers (ARBs), $\beta$ - or $\alpha$-receptor blockers, calcium antagonists, statins, or aspirin, was recorded. Pre-existing CVD was evaluated. CVD in our study included coronary artery disease (angina pectoris and myocardial infarction), arrhythmia, congestive heart failure, and cerebrovascular disease.

Glomerular filtration rate was estimated (eGFR) by the simplified Modified Diet in Renal Disease (MDRD) formula.

\section{MetS definition}

MetS was defined according to the National Cholesterol Education Program-Adult Treatment Panel III ${ }^{18-20}$ when patients had at least three of the following five criteria: (1) waist circumference $>90 \mathrm{~cm}$ or $80 \mathrm{~cm}$ for men and women, respectively; (2) systolic blood pressure $>130 \mathrm{mmHg}$ and/or diastolic blood pressure $>85 \mathrm{mmHg}$; (3) serum triglyceride (TG) levels $>1.69 \mathrm{mmol} / \mathrm{L}(150 \mathrm{mg} / \mathrm{dL})$; (4) serum high-density lipoprotein (HDL) cholesterol $<1.04 \mathrm{mmol} / \mathrm{L}$ (40 mg/dL) in men and $<1.30 \mathrm{mmol} / \mathrm{L}(50 \mathrm{mg} / \mathrm{dL})$ in women; and (5) fasting plasma glucose $>5.6 \mathrm{mmol} / \mathrm{L}$ $(100 \mathrm{mg} / \mathrm{dL})$.

\section{Laboratory methods}

Blood samples were obtained in the early morning after the subjects had fasted for at least 8 hours. Biochemical indices, such as fasting glucose, total cholesterol, TG, HDL cholestrol, low-density lipotrotein (LDL) cholesterol, blood urea nitrogen, serum creatinine, calcium, and phosphate, were determined using standard methods with the Olympus AU5400 chemistry immunoanalyzer (Olympus, Tokyo, Japan).

\section{Flow-mediated dilatation}

Noninvasive endothelium-dependent FMD was measured in each subject after they took a 10-minute rest in a supine position with an empty abdomen. The right brachial artery image was recorded in a longitudinal section $15 \mathrm{~cm}$ above the antecubital fossa using a $7 \mathrm{MHz}$ linear array transducer and a standard Acuson 128XP/10 system (Siemens, Erlangen, Germany). Baseline brachial artery diameter was measured using an automated wall tracking system (Sonoline G50, Siemens), which utilizes movement in the radiofrequency amplitude peaks over the cardiac cycle to identify the arterial walls. ${ }^{11,21}$ A pneumatic cuff was then inflated to $50 \mathrm{mmHg}$ above systolic blood pressure on the forearm for 5 minutes to induce a reactive hyperemia. Cuff deflation resulted in increased flow through the brachial artery, stimulating endothelial-dependent dilatation. The percentage of the change in brachial artery diameter 1 minute after cuff release to baseline diameter was recorded as FMD. Our coefficient of variation of FMD measurement in our study was $1.1 \% \pm 0.2 \%$ intra-assay and $4.5 \% \pm 1.0 \%$ interassay.

\section{Statistical analysis}

Statistical analysis was performed using SPSS software (v 16.0; SPSS Inc, Chicago, IL). Continuous variables are expressed as means \pm standard deviation, and categorical variables are expressed as percentages. Independent sample Student's $t$-test or one-way analysis of variance were used to compare two groups 
or multiple groups of normally distributed continuous variables, and the Mann-Whitney $U$ test was used for non-normally distributed variables. For comparison of categorical variables in two groups, such as the proportion of gender distribution, the $\chi^{2}$ test was employed. Pearson's correlation analysis was performed to explore the possible relationship between FMD and other continuous variables (Spearman's correlation analysis was performed if they were abnormal distributed variables). Multiple linear regression analysis was performed among those significant variables in Pearson's correlation analysis. A value of $P<0.05$ was taken as statistically significant.

\section{Results}

\section{Patients' clinical characteristics}

Demographic data of the study population are listed in Table 1. Of the 161 patients, 78 were male and 83 were female. Mean age was $65.58 \pm 14.57$ years. The etiologies of CKD were glomerulonephritis (29.2\%), diabetes mellitus (13.7\%), hypertension (20.5\%), and others (21.7\%).

\section{Comparison of clinical and laboratory data of patients with and without MetS}

Thirty-seven percent of the patients displayed MetS according to the National Cholesterol Education Program-Adult Treatment Panel III. As there was no significant difference in MetS and non-MetS between male and female patients, patients were grouped into two groups according to whether or not they had MetS. As is shown in Table 2, patients in the first group (those with MetS) had an expected higher weight and waist circumference than patients in the second group (both $P=0.000$ ). Also, primary renal diagnosis of diabetic nephropathy was more frequent $(P=0.008)$, TG $(P=0.000)$ and glucose $(P=0.000)$ were higher, and HDL cholesterol $(P=0.001)$ differed among the two groups, being lower in the MetS group compared with in the non-MetS group. There were no significant differences between the two groups with regard to blood pressure, MDRD stage, total cholesterol, calcium, phosphate, LDL, blood urea nitrogen, and serum creatinine. Notably, FMD was significantly lower in the group of patients with MetS than in the group without MetS $(7.65 \% \pm 3.93 \%$ vs $9.99 \% \pm 5.54 \%, z=-2.46$, $P=0.014$.). We found a higher total defined daily dose of antihypertensive medication in patients with MetS than in patients without MetS, but without significant difference.

\section{Association between FMD and other variables}

Pearson's correlation analysis between FMD and other variables is shown in Table 3. A significant negative correlation
Table I Demographic characteristics of the study population

\begin{tabular}{ll}
\hline & $\begin{array}{l}\mathbf{N} \pm \text { SD or number } \\
\text { and percentage }\end{array}$ \\
\hline Patients (number) & $16 \mathrm{I}$ \\
Male/female (ratio) & $78 / 83$ \\
Age (mean \pm SD), years & $65.58 \pm 14.57$ \\
Weight (mean \pm SD), kg & $65.56 \pm 11.99$ \\
Waist circumference (mean \pm SD), cm & $82.44 \pm 11.90$ \\
Primary diagnosis (\%) & \\
Chronic glomerulonephritis & 29.2 \\
Diabetes mellitus & 13.7 \\
Interstitial nephritis & 14.9 \\
Hypertension & 20.5 \\
Others & 21.7 \\
Systolic BP (mean \pm SD), mmHg & $117.75 \pm 22.83$ \\
Diastolic BP (mean \pm SD), mmHg & $70.36 \pm 14.17$ \\
MDRD stage, \% & \\
CKD stage I & 1.9 \\
CKD stage II & $1 \mathrm{I} .8$ \\
CKD stage III & 47.8 \\
CKD stage IV & 28.0 \\
CKD stage V & 10.6 \\
Pre-existing cardiovascular disease (\%) & 14 \\
Total DDD of antihypertensive drug & $1.45 \pm 1.22$ \\
ACEI DDD & $0.27 \pm 0.7 \mathrm{I}$ \\
ARB DDD & $0.32 \pm 0.57$ \\
Calcium antagonist DDD & $0.56 \pm 0.74$ \\
$\beta$-receptor blocker & $0.08 \pm 0.185$ \\
A-receptor blocker & $0.05 \pm 0.149$ \\
\hline Ab & \\
\hline
\end{tabular}

Abbreviations: $A C E l$, angiotensin-converting enzyme inhibitor; $A R B$, angiotensin receptor blocker; BP, blood pressure; CKD, chronic kidney disease; DDD, defined daily dose; MDRD, Modified Diet in Renal Disease; SD, standard deviation.

was found between FMD and waist circumference in women ( $r=-0.223, P=0.03)$ but not in men $(r=-0.055, P=0.625)$; moreover, FMD was inversely associated with fasting glucose $(r=-0.254, P=0.001)$ (Table 3$)$ and 24-hour urine protein excretion (Spearman correlation analysis $r=-0.204$, $P=0.011$ ), and FMD was also weakly correlated with serum calcium. FMD was higher in nondiabetic patients than in diabetic CKD patients $(10.07 \% \pm 5.48 \%$ vs $7.12 \% \pm 3.60 \%$, $P=0.000)$ and was also higher $(10.07 \% \pm 5.48 \%$ vs $6.97 \% \pm 3.95 \%, P=0.009)$ in nondiabetic patients than in high-fasting glucose patients without diabetes (fasting glucose $>6.1 \mathrm{mmol} / \mathrm{L}$ but $<7.0 \mathrm{mmol} / \mathrm{L}$ ), but there was no significant difference in FMD between diabetic and high-fasting glucose patients. There was no significant difference in patients' FMD between those with pre-existing CVD and those without CVD. No correlation was observed between FMD and age, blood pressure, serum cholesterol, TG, phosphate, uric acid, HDL cholesterol, LDL cholesterol, serum creatinine, and blood urea nitrogen (Table 3). We did not find an effect of drug agents such as ACEIs/ARBs, 
Table 2 Clinical and laboratory data of patients with and without metabolic syndrome

\begin{tabular}{|c|c|c|c|}
\hline & Metabolic syndrome $(n=59)$ & No metabolic syndrome $(n=102)$ & $P$ value \\
\hline Age, years & $66.34 \pm 15.08$ & $61.99 \pm 15.20$ & 0.068 \\
\hline Male/female & $26 / 33$ & $52 / 50$ & 0.398 \\
\hline Weight (kg) & $72.41 \pm 11.30$ & $61.60 \pm 10.54$ & 0.000 \\
\hline Waist circumference $(\mathrm{cm})$ & $91.01 \pm 8.34$ & $77.48 \pm 10.58$ & 0.000 \\
\hline Systolic BP (mmHg) & || $7.54 \pm 24.4 \mid$ & $117.87 \pm 21.99$ & 0.907 \\
\hline Diastolic BP (mmHg) & $71.02 \pm 15.36$ & $69.98 \pm 13.50$ & 0.694 \\
\hline \multicolumn{4}{|l|}{ Primary diagnosis $(\mathrm{n})(\%)$} \\
\hline Chronic glomerulonephritis & $14(23.7)$ & $33(32.4)$ & 0.283 \\
\hline Diabetes mellitus & 14 (23.7) & $8(7.8)$ & 0.008 \\
\hline Interstitial nephritis & $10(16.9)$ & 14 (I3.7) & 0.648 \\
\hline Hypertension & $13(22.0)$ & $20(19.6)$ & 0.840 \\
\hline \multicolumn{4}{|l|}{ MDRD stage (n) (\%) } \\
\hline CKD stage I & $0(0)$ & $3(2.9)$ & 0.299 \\
\hline CKD stage II & $7(11.9)$ & $12(11.8)$ & 1.000 \\
\hline CKD stage III & $30(50.8)$ & $47(46.1)$ & 0.624 \\
\hline CKD stage IV & $18(30.5)$ & $27(26.5)$ & 0.590 \\
\hline CKD stage $\mathrm{V}$ & $4(6.8)$ & $13(12.7)$ & 0.294 \\
\hline Total cholesterol $(\mathrm{mmol} / \mathrm{L})$ & $5.43 \pm 1.47$ & $5.14 \pm 1.13$ & 0.132 \\
\hline Triglycerides (mmol/L) & $2.96 \pm 1.95$ & $1.66 \pm 0.84$ & 0.000 \\
\hline Calcium (mmol/L) & $2.26 \pm 0.11$ & $2.25 \pm 0.22$ & 0.652 \\
\hline Phosphate (mmol/L) & $1.29 \pm 0.28$ & $1.34 \pm 0.24$ & 0.232 \\
\hline HDL cholesterol (mmol/L) & $1.32 \pm 0.80$ & $1.43 \pm 0.40$ & 0.001 \\
\hline LDL cholesterol (mmol/L) & $3.29 \pm 1.13$ & $3.04 \pm 0.96$ & 0.350 \\
\hline Fasting glucose (mmol/L) & $6.34 \pm 1.58$ & $5.50 \pm 1.13$ & 0.000 \\
\hline Uric acid $(\mu \mathrm{mol} / \mathrm{L})$ & $412.99 \pm 116.09$ & $349.91 \pm 112.81$ & 0.334 \\
\hline Serum creatinine $(\mu \mathrm{mol} / \mathrm{L})$ & $175.32 \pm 70.82$ & $200.56 \pm 124.49$ & 0.987 \\
\hline Blood urea nitrogen $(\mathrm{mmol} / \mathrm{L})$ & $|0.24 \pm 3.6|$ & $1 \mathrm{I} .78 \pm 6.66$ & 0.593 \\
\hline 24 -hour urine proteinuria excretion ( $g /$ day) & $1.24 \pm 1.84$ & $0.94 \pm 1.20$ & 0.393 \\
\hline Pre-existing cardiovascular disease (\%) & 16.7 & 11.0 & 0.404 \\
\hline Total DDD of antihypertensive drug & $1.78 \pm 1.36$ & $1.25 \pm 1.1$ & 0.969 \\
\hline FMD (\%) & $7.65 \pm 3.93$ & $9.99 \pm 5.54$ & 0.012 \\
\hline
\end{tabular}

Abbreviations: BP, blood pressure; CKD, chronic kidney disease; DDD, defined daily dose; FMD, flow-mediated dilatation; HDL, high-density lipoprotein; LDL, low-density lipoprotein; MDRD, Modified Diet in Renal Disease.

$\beta$ - or $\alpha$-blockers, Ca antagonists, statins, or aspirin on FMD determination (data not shown).

\section{Multiple linear regression analysis}

Multiple linear regression analysis showed that only fasting blood glucose was an independently associated factor for FMD (Table 4).

\section{Discussion}

More than half of the mortality for CKD patients is attributable to CVD. The true impact of CVD on outcomes for dialysis patients is better illustrated by the rate of death from CVD, which is 10-20 times higher than in the general population. ${ }^{22}$ Epidemiologic studies have shown an increased risk of cardiovascular mortality in the general population with MetS. ${ }^{23,24}$ The MetS is thought to be an important contributor to CVD among these patients and has become a major public health challenge around the world. ${ }^{25,26}$ How MetS increases
Table 3 Correlation analysis between flow-mediated dilatation and other variables

\begin{tabular}{lll}
\hline Variables & Correlation coefficient & $\boldsymbol{P}$ value \\
\hline Age & -0.086 & 0.227 \\
Systolic BP & 0.005 & 0.953 \\
Diastolic BP & 0.058 & 0.469 \\
UA & 0.033 & 0.674 \\
24-hour urine protein excretion & -0.204 & $0.01 I^{*}$ \\
Cr & 0.124 & 0.177 \\
BUN & 0.118 & 0.137 \\
eGFR & -0.002 & 0.984 \\
Fasting glucose & -0.254 & $0.00 I^{*}$ \\
Triglycerides & -0.105 & 0.186 \\
Total cholesterol & 0.030 & 0.708 \\
HDL-C & -0.04 I & 0.602 \\
LDL-C & $0.06 I$ & 0.441 \\
Calcium & 0.173 & $0.049 *$ \\
Phosphate & 0.042 & 0.594 \\
\hline Not & &
\end{tabular}

Note: *Significant at $P<0.05$.

Abbreviations: BP, blood pressure; BUN, blood urea nitrogen; $\mathrm{Cr}$, creatinine; eGFR, estimated glomerular filtration rate; HDL-C, high-density lipoprotein cholesterol; LDL-C, low-density lipoprotein cholesterol; UA, uric acid. 
Table 4 Coefficients of multiple regression analysis (dependent variable flow-mediated dilatation [\%])

\begin{tabular}{lllllll}
\hline & $\begin{array}{l}\text { Unstandardized } \\
\text { coefficients }\end{array}$ & SE & $\begin{array}{l}\text { Standardized } \\
\text { coefficients }\end{array}$ & t & P value & $\mathbf{9 5 \%} \mathbf{C l}$ \\
\hline Constant & 11.56 & 1.29 & & 8.95 & 0.000 & 9.01 to 14.11 \\
Fasting glucose & -0.44 & 0.20 & -0.18 & -2.17 & 0.03 & -0.8 to -0.04 \\
Waist circumstance & -0.10 & & & -1.17 & 0.24 & \\
Serum calcium & -0.03 & & & -0.33 & 0.74 & \\
\hline
\end{tabular}

Abbreviations: $\mathrm{Cl}$, confidence interval; SE, standard error.

the risk of CVD is not completely understood. Previous studies suggest that abnormal metabolism, associated with end-stage renal disease per se or with a pre-existing MetS, could also be an important driver of endothelial dysfunction and CVD.

Many previous studies had shown that FMD may reflect endothelial function. Endothelial dysfunction is both an early marker of vascular disease and a facilitative process in the development of atherosclerosis in the general population and patients with CKD. The Northern Manhattan Study (NOMAS) ${ }^{27}$ has shown that hazard ratios for cardiovascular events were $43 \%$ greater in those with MetS and 24\% greater in those with endothelial dysfunction than in those with neither, respectively, but there was no significant difference in patients' FMD whether or not they had pre-existing CVD in our current study. It might be because the role of pre-existing CVD in endothelial dysfunction of CKD patients was less than with MetS or its components.

All the components of MetS can individually impair endothelial function. Several studies have shown that both hypertension ${ }^{28}$ and diabetes type 2 are associated with endothelial dysfunction in the general population. ${ }^{29}$ Experimental glucose loading also impairs endothelial function, ${ }^{30}$ and abdominal obesity is associated with endothelial dysfunction. ${ }^{31}$ Impaired endothelial function was also reported in patients with low levels of HDL cholesterol. ${ }^{32}$ Reduced endothelium-dependent vasodilation (EDV) was also reported in patients with elevated TG levels. ${ }^{33}$

Regarding the contribution of each MetS component in the pathogenesis of endothelial dysfunction in patients with MetS, a large study $(\mathrm{n}=1016)$ by Lind reported that TG levels, waist circumference, and diastolic BP independently predicted EDV. ${ }^{34}$ Waist circumference was more strongly related to endothelial dysfunction followed by TG levels and diastolic blood pressure. ${ }^{34}$ In other reports, only HDL cholesterol levels ${ }^{35}$ were independently associated with reduced FMD in patients with MetS.

In our study, FMD was significantly lower in the group of patients with MetS than in the group of patients without MetS, indicating that MetS might be associated with endothelial dysfunction in patients with CKD. Moreover, patients with components of MetS, such as hyperglycemia (higher fasting blood glucose) and abdominal obesity in female CKD patients, correlated negatively with FMD, whereas other components, such as blood pressure, HDL cholesterol, and TG levels, did not correlate with FMD Fasting blood glucose level was the only independently associated factor with endothelial dysfunction, but only $13.7 \%$ of patients in our study were diabetic. The most common cause of CKD in China is glomerulonephritis, and many diabetic patients were followed up by an endocrine doctor. Only those patients with severe renal dysfunction were referred to a nephrologist.

We did not find the effect of blood pressure on FMD in our current study. This might have resulted from the patients' blood pressure being controlled (mean systolic blood pressure was $117.75 \pm 22 \mathrm{mmHg}$ ), and most of them received ACEIs/ARBs or calcium antagonist combinations with ACEIs/ARBs, so it is difficult to judge the effect of the antihypertensive drug category on endothelial dysfunction.

Some reports showed that serum uric acid levels are raised in MetS. ${ }^{36}$ Elevated serum uric acid levels appear to be associated with endothelial dysfunction and may play a role in the increased vascular risk of patients with MetS in the general population. ${ }^{36}$ In contrast to these results, we did not find that serum uric acid levels were associated with FMD, neither did we find that serum uric acid levels were significantly raised in CKD patients with MetS compared with those without MetS.

Our results did not show that there was any difference in patients with a different number of MetS components (data not shown).

Our results showed that FMD had no relationship with eGFR, indicating that endothelial dysfunction exists in early CKD stages, which may be a marker predicting CVD events in later stages of CKD. On the other hand, the eGFR by MDRD formula is not precisely for all stages of CKD. As previous reported, ${ }^{37}$ we also found that proteinuria was inversely associated with FMD. 
A few limitations remain in our study. The cross-sectional character of the present study cannot provide proof of direct causality between MetS components and FMD. Currently, we are performing a prospective, controlled, interventional study to explore the impact of management of the components of MetS (such as weight loss, control of blood glucose) on FMD in patients with CKD.

In summary, we showed that MetS and some components of MetS (fasting blood glucose and waist circumference in women) are closely associated with decreased FMD in CKD patients.

\section{Acknowledgment}

This study was supported by a Peking University Third Hospital Key Program Grant to Aihua Zhang (YZZ05-5-34).

\section{Disclosures}

The authors report no conflicts of interest. The authors alone are responsible for the content and writing of the paper.

\section{References}

1. Eckel RH, Grundy SM, Zimmet PZ. The metabolic syndrome. Lancet. 2005;365:1415-1428.

2. Chew GT, Khee GS, Watts GF. Revisiting the metabolic syndrome. Med J Aust. 2006;185:445-449.

3. Fulop T, Tessier D, Carpentier A. The metabolic syndrome. Pathol Biol. 2006;54:375-386.

4. Magliano DJ, Shaw JE, Zimmet PZ. How to best define the metabolic syndrome. Ann Med. 2006;38:34-41.

5. Wilson PW, D'Agostino RB, Parise H. Metabolic syndrome as a precursor of cardiovascular disease and type 2 diabetes mellitus. Circulation. 2005;112:3066-3072.

6. Malik S, Wong ND, Franklin SS. Impact of the metabolic syndrome MetS on mortality from coronary heart disease, cardiovascular disease, and all causes in United States adults. Circulation. 2004;110:1245-1250.

7. Benetos A, Laurent S, Asmar RG, Lacolley P. Large artery stiffness in hypertension. J Hypertens Suppl. 1997;15:S89-S97.

8. Brooks BA, Molyneaux LM, Yue DK. Augmentation of central arterial pressure in type 2 diabetes. Diabet Med. 2001;18:374-380.

9. Ferreira I, Snijder MB, Twisk JW, et al. Central fat mass versus peripheral fat and lean mass: opposite (adverse versus favorable) associations with arterial stiffness? The Amsterdam Growth and Health Longitudinal Study. J Clin Endocrinol Metab. 2004;89:2632-2639.

10. Sutton-Tyrrell K, Newman A, Simonsick EM, et al. Aortic stiffness is associated with visceral adiposity in older adults enrolled in the study of health, aging, and body composition. Hypertension. 2001;38:429-433.

11. Corretti MC, Anderson TJ, Benjamin EJ. Guidelines for the ultrasound assessment of endothelial-dependent flow-mediated vasodilation of the brachial artery: a report of the International Brachial Artery Reactivity Task Force. J Am Coll Cardiol. 2002;39:257-265.

12. Gokce N, Keaney JF Jr, Hunter LM. Predictive value of noninvasively determined endothelial dysfunction for long-term cardiovascular events in patients with peripheral vascular disease. J Am Coll Cardiol. 2003;41:1769-1775.
13. Vita JA, Keaney JF Jr. Endothelial function: a barometer for cardiovascular risk? Circulation. 2002;106:640-642.

14. Hsueh WA, Lyon CJ, Quinones MJ. Insulin resistance and the endothelium. Am J Med. 2004;117:109-117.

15. London GM, Marchais SJ, Guerin AP, Metivier F. Arteriosclerosis, vascular calcifications and cardiovascular disease in uremia. Curr Opin Nephrol Hypertens. 2005;14:525-531.

16. Johnson DW, Armstrong K, Campbell SB, et al. Metabolic syndrome in severe chronic kidney disease: prevalence, predictors, prognostic significance and effects of risk factor modification. Nephrology (Carlton). 2007;12:391-398.

17. Jabary NS, Herrero AM, Gonzalez JA. The use of antihypertensive therapy in Spain (1986-1994). Am J Hypertens. 2000;13:607-610.

18. Expert Panel on Detection, Evaluation, and Treatment of High Blood Cholesterol in Adults. Executive summary of the third report of the National Cholesterol Education Program (NCEP) Expert Panel on Detection, Evaluation, and Treatment of High Blood Cholesterol in Adults (Adult Treatment Panel III). JAMA. 2001;285:2486-2497.

19. Grundy SM, Brewer HB Jr, Cleeman JI, Smith SC Jr, Lenfant C. Definition of metabolic syndrome: report of the National Heart, Lung, and Blood Institute/American Heart Association conference on scientific issues related to definition. Circulation. 2004;109:433-438.

20. Grundy SM, Cleeman JI, Daniels SR, et al. Diagnosis and management of the metabolic syndrome: an American Heart Association/National Heart, Lung, and Blood Institute scientific statement. Circulation. 2005;112:2735-2752.

21. Celermajer DS, Sorensen KE, Gooch VM, et al. Non-invasive detection of endothelial dysfunction in children and adults at risk of atherosclerosis. Lancet. 1992;340:1111-1115.

22. Foley RN, Parfrey PS, Sarnak MJ. Epidemiology of cardiovascular disease in chronic renal disease. J Am Soc Nephrol. 1998;9:S16-S23.

23. Dekker JM, Girman C, Rhodes T. Metabolic syndrome MetS and 10-year cardiovascular disease risk in the Hoorn Study. Circulation. 2005;112:666-673.

24. Scuteri A, Najjar SS, Morrell CH. The metabolic syndrome MetS in older individuals: prevalence and prediction of cardiovascular events: the Cardiovascular Health Study. Diabetes Care. 2005;28:882-887.

25. Alberti KG, Zimmet P, Shaw J. Metabolic syndrome - a new worldwide definition. A Consensus Statement from the International Diabetes Federation. Diabet Med. 2006;23:469-480.

26. Grundy SM. Metabolic syndrome: connecting and reconciling cardiovascular and diabetes worlds. J Am Coll Cardiol. 2006;47:1093-1100.

27. Suzuki T, Hirata K, Elkind MS, et al. Metabolic syndrome, endothelial dysfunction, and risk of cardiovascular events: the Northern Manhattan Study (NOMAS). Am Heart J. 2008;156:405-410.

28. Benjamin EJ, Larson MG, Keyes MJ, et al. Clinical correlates and heritability of flow-mediated dilation in the community: the Framingham Heart Study. Circulation. 2004;109:613-619.

29. McVeigh GE, Brennan GM, Johnston GD, et al. Impaired endotheliumdependent and independent vasodilation in patients with type 2 (noninsulin-dependent) diabetes mellitus. Diabetologia. 1992;35:771-776.

30. Beckman JA, Goldfine AB, Gordon MB, Creager MA. Ascorbate restores endothelium-dependent vasodilation impaired by acute hyperglycemia in humans. Circulation. 2001;103:1618-1623.

31. Brook RD, Bard RL, Rubenfire M, Ridker PM, Rajagopalan S. Usefulness of visceral obesity (waist/hip ratio) in predicting vascular endothelial function in healthy overweight adults. Am J Cardiol. 2001;88:1264-1269.

32. Lupattelli G, Lombardini R, Schillaci G, et al. Flow-mediated vasoactivity and circulating adhesion molecules in hypertriglyceridemia: association with small, dense LDL cholesterol particles. Am Heart J. 2000;140:521-526.

33. Kuvin JT, Patel AR, Sidhu M, et al. Relation between high-density lipoprotein cholesterol and peripheral vasomotor function. Am J Cardiol. 2003;92:275-279. 
34. Lind L. Endothelium-dependent vasodilation, insulin resistance and the metabolic syndrome in an elderly cohort: the Prospective Investigation of the Vasculature in Uppsala Seniors (PIVUS) study. Atherosclerosis. 2008;196:795-802.

35. Dell'Omo G, Penno G, Pucci L, Mariani M, Del PS, Pedrinelli R. Abnormal capillary permeability and endothelial dysfunction in hypertension with comorbid metabolic syndrome. Atherosclerosis. 2004; 72: 383-389.
36. Tomiyama H, Higashi Y, Takase B, et al. Relationships among hyperuricemia, metabolic syndrome, and endothelial function. Am J Hypertens. 2011;24:770-774.

37. Sonmez A, Yilmaz MI, Saglam M, et al. The relationship between hemoglobin levels and endothelial functions in diabetes mellitus. Clin J Am Soc Nephrol. 2009;5:45-50.

\section{Publish your work in this journal}

Vascular Health and Risk Management is an international, peerreviewed journal of therapeutics and risk management, focusing on concise rapid reporting of clinical studies on the processes involved in the maintenance of vascular health; the monitoring, prevention and treatment of vascular disease and its sequelae; and the involvement of metabolic disorders, particularly diabetes. This journal is indexed on PubMed Central and MedLine. The manuscript management system is completely online and includes a very quick and fair peer-review system, which is all easy to use. Visit http://www.dovepress.com/ testimonials.php to read real quotes from published authors.

Submit your manuscript here: http://www.dovepress.com/vascular-health-and-risk-management-journal 